\title{
Experimental Study of the Compressive Strength of Multi-Coat Renders
}

\author{
António Soares ${ }^{a}$, Inês Flores-Colen ${ }^{a}$,Jorge de Brito ${ }^{a *}$ \\ ${ }^{a}$ Departamento de Engenharia Civil, Arquitectura e Georrecursos, Investigação e Inovação em \\ Engenharia Civil para a Sustentabilidade, Universidade Técnica de Lisboa, Av. Rovisco Pais, \\ 1049-001, Lisboa, Portugal
}

Received: July 25, 2016; Revised: May 09, 2017; Accepted: June 20, 2017

\begin{abstract}
Mortar compressive strength is a standard requirement and should be tested on stand-ardized specimens with a $40 \mathrm{~mm}$ square cross section. However, these specimens with only one type of mortar (base-coat layer) do not represent the render traditionally ap-plied to façades since that must consist of at least two layers (base coat and top coat, with different formulations and thicknesses). The in-service compressive strength of the render is therefore currently evaluated on-site by means of indirect methods (e.g. pendulum rebound hammer or ultrasound). Addi-tionally, studies on evaluating the compressive strength in multilayer coating systems, based on the laboratory analysis of the mortar samples collected in situ, are few and far between. This paper intends to discuss the method of assessing in-service compressive strength of applied multi-coat renders systems, by testing cores collected after pull-off tests. Therefore, 422 compressive strength tests were carried out to establish a relation-ship between the compressive strength of single- and multi-coat renders and identify which render layers influence the system's compressive strength. The experimental cam-paign results have shown the great influence of the top coat on multi-coat renders' per-formance and the feasibility of studying the in-service compressive strength after a pull-off test; and also allowed identifying relevant parameters to evaluate the strength of samples collected in-situ.
\end{abstract}

Keywords: Coating mortars, in-service performance, compressive strength, cores

\section{Introduction}

The study of in-service compressive strength is widely used in the assessment of concrete structures under actual service conditions. In these in-situ evaluations, several cores are drilled and collected for compression strength testing in the lab, according to EN $13791^{1}$. Also the concrete core strength results are usually correlated with other indirect methods, such as rebound hammer index and ultrasonic pulse velocity.

The concept of estimating actual concrete in-situ strength relative to cubic specimens is employed when the values of the strength of cores are used for structural calculation or to give the strength of an existing structure ${ }^{2}$.

When comparing standard specimens produced in the lab with the same concrete as that used in construction works, their strength will differ from that determined in situ, even if we consider that it is possible to obtain perfect cores of the same size as the test pieces. This is because of differences in compacting and curing ${ }^{2}$. Therefore, the study of in-service compressive strength should be performed on cores collected onsite.

In the case of renders, the study of in-service compressive strength can help the diagnosis of the causes of several render anomalies related with high susceptibility to cracks; low resistance to the impact loads; inadequate specification

*e-mail: jb@civil.ist.utl.pt in terms of in-service loads; mechanical incompatibility of render and its support, among others. This property is a standard requirement in EN 998- $1^{3}$ and under EN 1015-114, the device used to test the compressive strength of mortars should have $40 \mathrm{~mm}$ square plates so as to obtain the strength of $40 \mathrm{~mm}$ cubic specimens. But these samples do not represent the render traditionally used on façades since, in accordance with EN 13914- $1^{5}$ and other national technical specifications suggested by the National Laboratory of Civil Engineering ${ }^{6}$, conventional render should be applied in at least two coats (base and top coats) and, sometimes by using a spatterdash coat for adherence purposes.

It is therefore hard to directly evaluate the compressive strength of multi-coat systems, and so indirect methods have been used, namely a pendulum rebound hammer or ultrasound on the walls surface.

Several authors have tried to discuss the direct assessment of compressive strength of lime-based renders applied on old buildings walls by carrying out tests on cores extracted from existing render systems. Válek and Veiga ${ }^{7}$ used irregular samples of historic mortars collected in-situ to study a simple adaptation of the method to determine the compressive strength recommended by the European Standard EN 1015$11^{4}$, to enable the compressive testing of irregular samples collected from coatings on old buildings. The adaptation involved applying a confining mortar with a 1:3 volumetric 
ratio (CEM II, 32.5: sand), on the two parallel sides of the irregular sample.

Magalhães and Veiga ${ }^{8}$ used reference samples measuring $20 \times 40 \times 80\left[\mathrm{~mm}^{3}\right]$, cut from pieces measuring $40 \times 40 \mathrm{x}$ $160\left[\mathrm{~mm}^{3}\right]$, to assess the method. Confining mortar was applied for the compression test. The compressive strength values obtained with these samples were compared with those of the standard test specimens, resulting in a good linear relationship $\left(\mathrm{R}^{2}>0.96\right)$; with this correlation the values of compressive strength obtained by the adapted method were higher than the ones obtained by the standard method. The higher compressive strength of the reference specimens (RSs) relative to the standard specimens (SSs) was explained by the greater carbonation of the RSs, which were tested over a 1-3 year period after the SSs were tested ${ }^{8}$. The authors concluded that the proposed method can make a valid contribution, albeit without furnishing rigorous results in absolute terms, to finding the mechanical strength of old buildings ${ }^{9}$.

Flores-Colen ${ }^{10}$ studied the in-service mechanical performance of cement-based traditional and pre-dosed renders, through in-situ and lab testing on collected samples. The research concluded that the pull-off technique, despite being destructive, is a probing method that can help the study of render mechanical parameters. The authors identify the benefits of studying the render cores after a pull-off test. Good correlations were obtained between adhesion results and compression tests $\left(\mathrm{R}^{2}>0.80\right)$. The compressive strength was studied in standard specimens and cores. Also, acceptable results allowed estimating the cores compressive strength in terms of ten times the stress in cohesive failure from the pull-off test $\left(\mathrm{R}^{2}>0.70\right)$. However, core compressive strength results obtained in the lab showed high variation coefficients $(\mathrm{CV}>25 \%)$ and needed further investigation.

Due to the thickness of the in-situ renders, it is also necessary to take into account the limitation this causes in the geometry of the sample (test piece) for the evaluation of the compressive strength of the render, since the compressive strength of products such as concrete and mortar is affected by the size of the test piece ${ }^{11-12}\left(\right.$ cited by $\left.^{13}\right)$, and it decreases in concrete through a power trend the more slender the test piece $^{14-15}$. A number of authors ${ }^{16-18}$, have recently studied the compressive strength of mortars and found that, as with concrete it increases as slenderness decreases through a power trend. Soares et al. ${ }^{18}$ further obtained for industrial and traditional renders a power trend relationship between compressive strength (as a function of the strength index) and slenderness (h/d) of the test piece $\left(0.60<\mathrm{R}^{2}<0.80\right)$, except for traditional multilayer render (spatterdash, base coat, top coat), making it difficult to directly compare the compressive strength of a multi-coat render with that of standard specimens.

In summary, there are very few studies on evaluating the compressive strength of multilayer coating systems through standard procedures. Given the possibility of evaluating compressive strength from multilayer cores, it is important to assess the relationship between the compressive strength of test pieces and cores from a single layer and a multilayer and to identify the coating layers that affect the compressive strength results.

Soares et al. ${ }^{19}$ studied (in the laboratory) the possibility of producing reference specimens with more than one layer (Figures 1 and 2), to represent conventional multi-coat cores of render, with the purpose of evaluating the compressive strength of cementitious mortars used in walls rendering. The author obtained good results, finding a linear relationship between compressive strength values for cores and reference specimens of traditional multilayer render close to $\mathrm{y}=\mathrm{x}$ (with a slope of $1 \pm 0.22$ ). The same author found that this relationship does not change significantly with age $\left(\mathrm{R}^{2}=\right.$ $0.86)$ when grouping the results of the all ages $(7,14,28$ and 90 days).
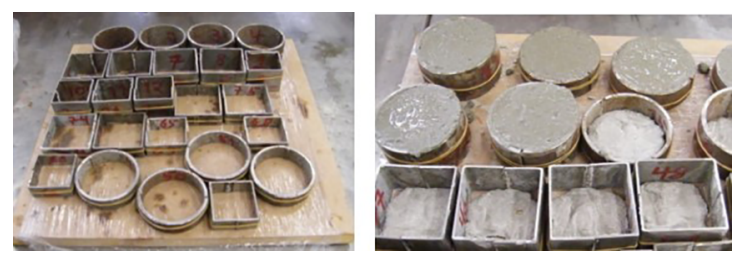

Figure 1. Steel moulds for reference specimens (left) and with base coat in fresh state and hardened spatterdash (right).
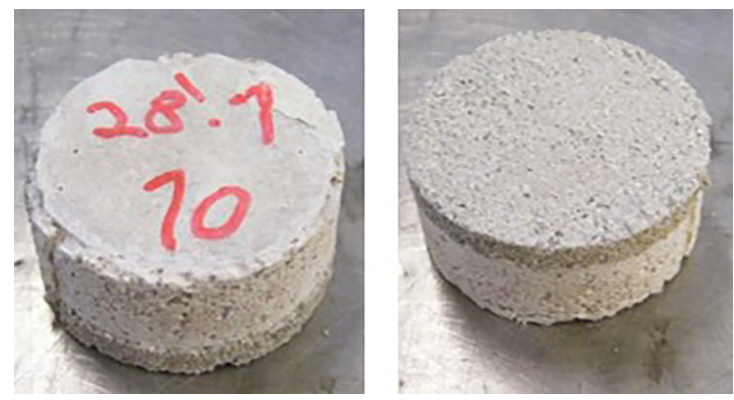

Figure 2. Final aspect of the circular reference specimens, with several layers.

The expected compressive strength of the render was characterized by standard specimens testing at 7, 14, 8 and 90 days. The results are given in Table 1 .

The cores were extracted from render applied to brick and tested, along with test pieces moulded in the laboratory (reference specimens), using the same constituents and having the same geometric characteristics as the cores.

This paper presents part of the research conducted and discusses the relationship between the compressive strength of conventional single-layer and multilayer cement-based render. The aim is to study the possibility of evaluating the mechanical strength of a core or specimen of render with several coats, using a single-coat specimen. 
Table 1. Compressive strength values measured in standardized specimens (SSs)

\begin{tabular}{|c|c|c|c|c|}
\hline Single-coat traditional render/base coat of multi-coat traditional $\mathrm{r}$ & & & & \\
\hline Age [days] & 7 & 14 & 28 & 90 \\
\hline Compressive strength of standardized specimens (SSs) $\left[\mathrm{N} / \mathrm{mm}^{2}\right]$ & 8,8 & 10,6 & 10,8 & 13,1 \\
\hline
\end{tabular}

The authors intend to identify the layers with more relevance to assess compressive strength of multilayer render cores and evaluate the feasibility of this method to evaluate directly the in-service compressive strength of existing renders, taking advantage of pull-off cores without resorting to complex methods such as finite elements analysis or computational models.

\section{Experimental Procedure}

Reference specimens (RSs) were produced and conventional multi-coat renders were applied to bricks (spatterdash, base coat and top coat). Control conventional renders were also produced (one coat with the same composition as the base coat of a multi-coat render). The relevant thicknesses are given in Table 2.

\subsection{Preparation of traditional render}

The traditional mortar was produced as prescribed in EN $196-1^{20}$, using river sand as aggregate and CEM II/B-L $32.5 \mathrm{~N}$ cement as binder.

The volumetric ratios used for each traditional render coat were as established in $\mathrm{LNEC}^{6}$, within the standards of good practice, as follows: spatterdash at 1:2; base coat 1:4, and top coat 1:4.5.

The water content of each formulation was determined based on the flow table test so as to arrive at a flow value of $175 \pm 10 \mathrm{~mm}$, per EN 1015-21 , apart for the spatterdash that had a fluid consistency. Table 2 shows the composition of each coat of the traditional render.

\subsection{Application of renders prepared in the laboratory}

The mortar for the traditional render was applied according to EN 13914-15. This standard does not allow for the waiting time required between applying each coat of render since this varies depending on the climatic conditions. We followed the suggestions in Gomes et al. ${ }^{6}$ for the 3 -coat traditional render, leaving an interval of 3 days between applying the spatterdash and the base coat, and 8 days between that and the top coat.

It should also be remembered that according to EN 13914-1 ${ }^{5}$ the spatterdash should not be regarded as a coat and should be ignored when measuring the total thickness of the coat. But according to $\mathrm{LNEC}^{18}$ it can contribute to compressive strength, and so for the purpose of this work it was applied evenly to the surface of the substrate and is deemed a layer for the evaluation of its influence on the compressive strength of the coating system.

The surface of the intermediate layers of the traditional multi-coat render was roughened to encourage better bonding between coats.

\subsection{Reference specimens (RSs)}

The reference specimens were prepared ${ }^{19}$, based on procedures for standard size specimens, using steel moulds (Figure 1, left, with inner measurements similar to those of the cores (Cs) obtained with the pull-off adhesion test (render applied on a brick).

The mortar was placed in the moulds and compacted by tapping from above with a trowel to achieve a uniform distribution of fresh mortar in the mould, and finally pressed to give a smooth finish. Figure 2 shows the final look of the circular cross section reference specimens.

In the reference specimens of multi-coat render the mould of the next layer was placed around the one already produced so that the bond between the coats would be continuous (Figure 1, right).

\subsection{Storage and curing of the specimens}

The specimens were stored and cured as described in EN $1015-11^{4}$ by being placed in a conditioned chamber at a temperature of $20 \pm 2{ }^{\circ} \mathrm{C}$ and relative humidity of $65 \pm$ $5 \%$. The samples were wrapped in polyethylene bags for the initial curing.

Because the top coat was applied 8 days after the base coat the samples were kept in the polyethylene bags for a further 3 days to ensure that this last layer was wet cured.

The initial curing of the different types of render studies was harmonized by keeping the single-coat traditional render samples in polyethylene bags for 11 days, except for the ones tested at 7 days, which were only kept in the bags for 4 days, since it would not be possible to carry out the whole pull-off adhesion test procedure and the subsequent compressive strength test.

\subsection{Compressive strength test}

The procedure established in EN 1015-11 ${ }^{4}$ was followed, using a Seidner Form+Test instrument (model 505/200/10 DM1), with a $200 \mathrm{kN}$ load cell, at a rate between $50 \mathrm{~N} / \mathrm{s}$ and $500 \mathrm{~N} / \mathrm{s}$.

Cores from the pull-off adhesion test in accordance with EN 1015-1222 were subjected to compression testing. In 
Table 2. Characteristics of the traditional render

\begin{tabular}{lcccc}
\hline Traditional render & & Spatterdash & Base coat & Top coat \\
\hline \multirow{3}{*}{ Thickness (mm) } & Type 1 & 3 & 15 & 6 \\
& Type 2 & 3 & 25 & 6 \\
& Type 5 & - & 15 & - \\
Volumetric ratio & Type 6 & - & 25 & - \\
Water/cement ratio & & $1: 2$ & $1: 4$ & $1: 4,5$ \\
\hline
\end{tabular}

Caption: Type 1 and Type 2 - multi-coat traditional renders Type 5 and Type 6 - control traditional renders with one coat.

addition to the $50 \mathrm{~mm}$ diameter circular cross section cores, $40 \mathrm{~mm}$ and $50 \mathrm{~mm}$ square cross section cores were also prepared, as suggested by RILEM ${ }^{23}$ for in-situ tests which recommends using square cross section cores with an area the same as the circular cross section ones.

Therefore, three $50 \mathrm{~mm}$ diameter circular cross section cores, two $40 \mathrm{~mm}$ square cross section cores and one 50 $\mathrm{mm}$ square cross section core were taken from each brick. RSs with similar characteristics to the Cs were also tested in compression, making a total of 422 valid tests, as detailed in Tables 3 to 5 . Some of the tests at 28 days were conducted using a Controls test device (model L11D2) with a $250 \mathrm{kN}$ load cell, at the same rate of loading, which may explain the lower values of compressive strength at 28 days of some specimens (relative to 14 days)

\subsection{Preparation of cores for the compressive strength test}

Before the cores could be used for the compressive strength test the steel disc and glue required for the pull-off adhesion test had to be removed without damaging the core.

The procedure described by Flores-Colen ${ }^{10}$ was used, whereby the glue is melted on an electric stove and the core is then detached from the disc by means of a spatula (Figure 3).

The irregularities on the cores' surface, caused by contact with the brick, were smoothed with a file in preparation for contact with the compression instrument's plates (Figure 4).

As the cross sectional area of the specimen was sometimes larger than that of the testing machine's plates, two steel plates with an area greater than the sample's surface area were used (Figure 5). They were placed on the ends of the core such that the compression force would be distributed over the entire area. These plates were used on all the cores to keep the test conditions identical. A steel wedge was used with the cores of reduced thickness (Figure 5) to adjust their height so that they would fit between the compression machine's plates.

\section{Presentation and Discussion of the Results}

The compressive strength values obtained for both the Cs and RSs were compared to see if is possible to evaluate the compressive strength of traditional multi-coat render through the value determined for a single-layer traditional render (made with the same mixture and being the same size as the base coat of the multi-coat render), i.e. a simple method without resorting to complex techniques or computational models.

\subsection{Compressive strength relationship between multi-coat and single-coat cores}

Analysis of the relationship between the strengths found for single- and multi-coat render cores showed that the relationship at 7 days has a slope of 0.87 (which is close to $y$ $=\mathrm{x}$ ), with a correlation coefficient $\mathrm{R}^{2}=0.76$ (Figure 6), while at other ages the slope increases, with correlation coefficients of the same order of magnitude. The slope increase is most marked at 28 days. However, the slope obtained with the 28 day results has a correlation coefficient $\mathrm{R}^{2} \approx 0.38$, which can be ascribed to the 3 highest compressive strength values at this age for the single-coat render coming from specimens whose slenderness $(\mathrm{h} / \mathrm{d})$ is between 0.33 and 0.37 , which are low slenderness values. According to Soares et al. ${ }^{18}$, these slenderness values result in high compressive strength values of considerable variability, and therefore not representative.

The traditional multi-coat render tested at 7 days was produced without a top coat, i.e. it comprised only spatterdash and base coat. This aspect of cores tested at age 7 days, plus the fact that a slope closest to $\mathrm{y}=\mathrm{x}$ was obtained at this age, with a good correlation coefficient $\left(\mathrm{R}^{2}=0.76\right.$ for cores and $\mathrm{R}^{2}=0.99$ for reference specimens), may indicate that the spatterdash coat (the render coat with the strongest cement ratio) does not exert a significant influence on compressive strength.

The relationship obtained by grouping all the ages shows a trend line in Figure 6 with a slope of 0.94 , which is close to $\mathrm{y}=\mathrm{x}$. However, this has $\mathrm{R}^{2} \approx 0.18$, so it cannot be representative of the various ages treated separately, which was expected since three-coat examples (aged 14, 28 and 90 days) are being combined with two-coat examples (aged 7 days, without top coat). When only three-coat examples are grouped together we find a considerable increase in the correlation coefficient up to $\mathrm{R}^{2} \approx 0.58$, which, even though it is not a high value, can be explained by the deviation from the trend line in the 28 day results with a low $\mathrm{R}^{2}$ value. 
Table 3. Number of compressive strength tests

\begin{tabular}{|c|c|c|c|c|c|c|c|}
\hline Type of render & $\begin{array}{l}\text { Type of } \\
\text { specimen }\end{array}$ & \multicolumn{3}{|c|}{ Multi-coat traditional render } & \multicolumn{3}{|c|}{ Single-coat traditional render } \\
\hline Core diameter/cross section & & D50 & L40 & L50 & D50 & L40 & L50 \\
\hline \multirow{2}{*}{ Tests } & $\mathrm{C}$ & 62 & 47 & 23 & 24 & 15 & 7 \\
\hline & RS & 47 & 55 & 48 & 31 & 34 & 29 \\
\hline \multirow{5}{*}{ Total } & $\mathrm{C}$ & & 132 & & & 46 & \\
\hline & $\mathrm{RS}$ & 150 & 94 & & & & \\
\hline & $\mathrm{C}$ & \multicolumn{6}{|c|}{178} \\
\hline & $\mathrm{RS}$ & \multicolumn{6}{|c|}{244} \\
\hline & & \multicolumn{6}{|c|}{422} \\
\hline
\end{tabular}

Caption: C - cores taken from render applied to brick; RS - reference specimens; D50 - $50 \mathrm{~mm}$ diameter circular cross section specimens; L40 - 40 mm square cross section specimens; L50 - 50 mm square cross section specimens.

Table 4. Average results and standard deviations of the compressive strength tests of cores

\begin{tabular}{|c|c|c|c|c|c|}
\hline Type of render & $\begin{array}{c}\text { Core diameter/cross } \\
\text { section }\end{array}$ & 7 days & 14 days & 28 days & 90 days \\
\hline \multirow{3}{*}{$\begin{array}{l}\text { Multi-coat } \\
\text { traditional render } \\
\text { (base coat with } 15 \\
\text { mm thickness) }\end{array}$} & D50 & $15.38( \pm 1.04)$ & $11.59( \pm 0.91)$ & $10.05( \pm 2.95)$ & $12.46( \pm 2.25)$ \\
\hline & L40 & $14.42( \pm 4.08)$ & $9.15( \pm 0.91)$ & $10.81( \pm 4.79)$ & $14.76( \pm 0.97)$ \\
\hline & L50 & $22.38( \pm 0.00)$ & $15.06( \pm 1.70)$ & $10.63( \pm 4.10)$ & - \\
\hline \multirow{3}{*}{$\begin{array}{l}\text { Multi-coat } \\
\text { traditional render } \\
\text { (base coat with } 25 \\
\text { mm thickness) }\end{array}$} & D50 & $10.55( \pm 1.08)$ & $8.01( \pm 1.90)$ & $9.54( \pm 1.71)$ & $9.34( \pm 0.87)$ \\
\hline & L40 & $8.57( \pm 0.77)$ & $8.00( \pm 0.93)$ & $8.00( \pm 2.68)$ & $10.68( \pm 1.55)$ \\
\hline & L50 & $12.00( \pm 0.25)$ & $11.83( \pm 3.46)$ & $10.48( \pm 1.59)$ & $12.28( \pm 2.06)$ \\
\hline \multirow{3}{*}{$\begin{array}{l}\text { Single-coat } \\
\text { traditional render } \\
\text { (with } 15 \mathrm{~mm} \\
\text { thickness) }\end{array}$} & D50 & $18.09( \pm 2.70)$ & $24.34( \pm 1.09)$ & $22.61( \pm 1.64)$ & $26.09( \pm 1.67)$ \\
\hline & L40 & $15.49( \pm 0.17)$ & $20.53( \pm 0.26)$ & $20.20( \pm 0.25)$ & $27.06( \pm 0.69)$ \\
\hline & L50 & $18.48( \pm 0.00)$ & $36.06( \pm 0.00)$ & $27.17( \pm 0.00)$ & - \\
\hline \multirow{3}{*}{$\begin{array}{l}\text { Single-coat } \\
\text { traditional render } \\
\text { (with } 25 \mathrm{~mm} \\
\text { thickness) }\end{array}$} & D50 & $8.41( \pm 0.67)$ & $12.31( \pm 3.21)$ & $10.97( \pm 0.37)$ & $11.72( \pm 1.74)$ \\
\hline & L40 & $8.28( \pm 0.50)$ & $11.04( \pm 0.37)$ & $10.25( \pm 0.00)$ & $14.15( \pm 040)$ \\
\hline & L50 & $9.66( \pm 0.00)$ & $14.85( \pm 0.00)$ & $11.49( \pm 0.00)$ & $16.38( \pm 0.00)$ \\
\hline Type of render & $\begin{array}{c}\text { Core diameter/cross } \\
\text { section }\end{array}$ & 7 days & 14 days & 28 days & 90 days \\
\hline \multirow{3}{*}{$\begin{array}{l}\text { Multi-coat } \\
\text { traditional render } \\
\text { (base coat with } 15 \\
\text { mm thickness) }\end{array}$} & D50 & $15.38( \pm 1.04)$ & $11.59( \pm 0.91)$ & $10.05( \pm 2.95)$ & $12.46( \pm 2.25)$ \\
\hline & L40 & $14.42( \pm 4.08)$ & $9.15( \pm 0.91)$ & $10.81( \pm 4.79)$ & $14.76( \pm 0.97)$ \\
\hline & L50 & $22.38( \pm 0.00)$ & $15.06( \pm 1.70)$ & $10.63( \pm 4.10)$ & - \\
\hline \multirow{3}{*}{$\begin{array}{l}\text { Multi-coat } \\
\text { traditional render } \\
\text { (base coat with } 25 \\
\text { mm thickness) }\end{array}$} & D50 & $10.55( \pm 1.08)$ & $8.01( \pm 1.90)$ & $9.54( \pm 1.71)$ & $9.34( \pm 0.87)$ \\
\hline & L40 & $8.57( \pm 0.77)$ & $8.00( \pm 0.93)$ & $8.00( \pm 2.68)$ & $10.68( \pm 1.55)$ \\
\hline & L50 & $12.00( \pm 0.25)$ & $11.83( \pm 3.46)$ & $10.48( \pm 1.59)$ & $12.28( \pm 2.06)$ \\
\hline \multirow{3}{*}{$\begin{array}{l}\text { Single-coat } \\
\text { traditional render } \\
\text { (with } 15 \mathrm{~mm} \\
\text { thickness) }\end{array}$} & D50 & $18.09( \pm 2.70)$ & $24.34( \pm 1.09)$ & $22.61( \pm 1.64)$ & $26.09( \pm 1.67)$ \\
\hline & L40 & $15.49( \pm 0.17)$ & $20.53( \pm 0.26)$ & $20.20( \pm 0.25)$ & $27.06( \pm 0.69)$ \\
\hline & L50 & $18.48( \pm 0.00)$ & $36.06( \pm 0.00)$ & $27.17( \pm 0.00)$ & - \\
\hline \multirow{3}{*}{$\begin{array}{l}\text { Single-coat } \\
\text { traditional render } \\
\text { (with } 25 \mathrm{~mm} \\
\text { thickness) }\end{array}$} & D50 & $8.41( \pm 0.67)$ & $12.31( \pm 3.21)$ & $10.97( \pm 0.37)$ & $11.72( \pm 1.74)$ \\
\hline & L40 & $8.28( \pm 0.50)$ & $11.04( \pm 0.37)$ & $10.25( \pm 0.00)$ & $14.15( \pm 040)$ \\
\hline & L50 & $9.66( \pm 0.00)$ & $14.85( \pm 0.00)$ & $11.49( \pm 0.00)$ & $16.38( \pm 0.00)$ \\
\hline
\end{tabular}

Caption: D50 - $50 \mathrm{~mm}$ diameter circular cross section specimens; L40 - $40 \mathrm{~mm}$ square cross section specimens; L50 - $50 \mathrm{~mm}$ square cross section specimens. 
Table 5. Average results and standard deviations of the compressive strength tests of reference specimens

\begin{tabular}{|c|c|c|c|c|c|}
\hline Type of render & $\begin{array}{l}\text { Reference } \\
\text { specimen diameter/ } \\
\text { cross section }\end{array}$ & 7 days & 14 days & 28 days & 90 days \\
\hline \multirow{3}{*}{$\begin{array}{l}\text { Multi-coat } \\
\text { traditional render } \\
\text { (base coat with } 15 \\
\text { mm thickness) }\end{array}$} & D50 & $19.78( \pm 1.51)$ & $15.27( \pm 1.20)$ & $13.42( \pm 1.65)$ & $18.69( \pm 1.01)$ \\
\hline & L40 & $12.50( \pm 0.50)$ & $9.13( \pm 0.51)$ & $9.17( \pm 1.16)$ & $12.08( \pm 1.52)$ \\
\hline & L50 & $17.63( \pm 2.06)$ & $13.61( \pm 0.46)$ & $12.16( \pm 1.18)$ & $16.97( \pm 0.85)$ \\
\hline \multirow{3}{*}{$\begin{array}{l}\text { Multi-coat } \\
\text { traditional render } \\
\text { (base coat with } 25 \\
\text { mm thickness) }\end{array}$} & D50 & $8.50( \pm 0.81)$ & $7.93( \pm 1.06)$ & $8.93( \pm 1.60)$ & $12.57( \pm 0.61)$ \\
\hline & L40 & $7.03( \pm 0.37)$ & $7.28( \pm 1.09)$ & $7.05( \pm 1.40)$ & $7.51( \pm 1.19)$ \\
\hline & L50 & $8.96( \pm 0.50)$ & $9.90( \pm 0.88)$ & $9.89( \pm 0.93)$ & $12.49( \pm 0.78)$ \\
\hline \multirow{3}{*}{$\begin{array}{l}\text { Single-coat } \\
\text { traditional render } \\
\text { (with } 15 \mathrm{~mm} \\
\text { thickness) }\end{array}$} & D50 & $24.18( \pm 0.77)$ & $28.86( \pm 1.93)$ & $20.28( \pm 1.14)$ & $25.96( \pm 3.23)$ \\
\hline & L40 & $15.07( \pm 0.93)$ & $15.64( \pm 0.96)$ & $11.68( \pm 1.86)$ & $16.79( \pm 3.07)$ \\
\hline & L50 & $20.50( \pm 2.11)$ & $24.97( \pm 0.00)$ & $18.04( \pm 0.63)$ & $26.17( \pm 2.61)$ \\
\hline \multirow{3}{*}{$\begin{array}{l}\text { Single-coat } \\
\text { traditional render } \\
\text { (with } 25 \mathrm{~mm} \\
\text { thickness) }\end{array}$} & D50 & $11.65( \pm 0.59)$ & $15.67( \pm 1.25)$ & $11.63( \pm 0.44)$ & $12.32( \pm 1.37)$ \\
\hline & L40 & $9.22( \pm 0.77)$ & $10.29( \pm 1.42)$ & $9.71( \pm 0.88)$ & $8.69( \pm 1.10)$ \\
\hline & L50 & $10.93( \pm 0.47)$ & $12.13( \pm 0.23)$ & $10.82( \pm 0.56)$ & $10.69( \pm 1.23)$ \\
\hline
\end{tabular}

Caption: D50 - $50 \mathrm{~mm}$ diameter circular cross section specimens; L40 - $40 \mathrm{~mm}$ square cross section specimens; L50 - $50 \mathrm{~mm}$ square cross section specimens.
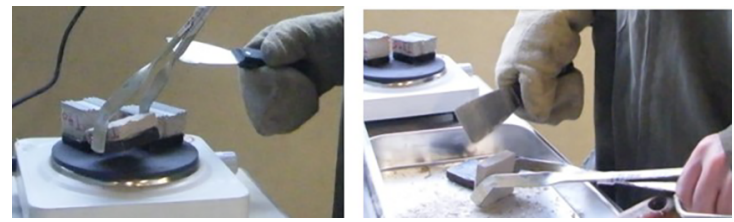

Figure 3. Heating the epoxy glue and removal of steel disc from the core.
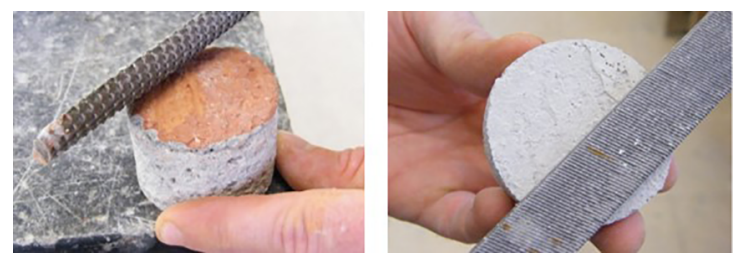

Figure 4. Rectification of surfaces of cores and reference specimens.

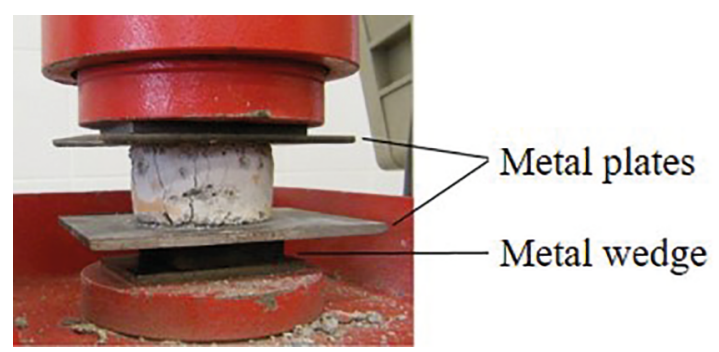

Figure 5. Compressive strength test using two steel plates and a steel wedge.

Thus, bearing in mind the slope $\mathrm{y}>\mathrm{x}$ observed in the comparison of single-coat traditional and multi-coat (spatterdash, base coat and top coat) render, it can be said that the multi-coat traditional render has lower strength than the single-coat traditional render (produced with the same product as the base coat of the multi-coat traditional render. This can be explained by the higher slenderness $(\mathrm{h} / \mathrm{d})$ of the multi-coat traditional render, or by it having a top coat that is produced with a lower cement ratio. Another reason for slope $y>x$ can be related to water suction by the substrate that can modify the internal structure and the density of mortars after application, affecting the coats' performance (e.g. spatterdash and base coat).

\subsection{Compressive strength relationship between multi-coat and single-coat reference specimens}

The relationship between reference specimens of traditional single-coat and multi-coat renders (Figure 7) shows good correlation coefficients $\left(\mathrm{R}^{2}\right)$ at each test age analysed individually, i.e. they are all above 0.8 , and the slopes are in most cases lower than those from the cores' analysis. The higher $\mathrm{R}^{2}$ values in the reference specimens may be related with the greater size precision that is achieved in moulding these specimens by comparison with the cores that result from the pull-off test, which may cause some variation in dimensions and non-visible damage, such as microcraking.

To better appreciate the difference in the ratios of each layer of the multi-coat traditional render, Figure 8 (left) shows a reference specimen (cross section) after the compressive strength test, where it is possible to see the different layers of the render. Note that the spatterdash coat is the most compact and the others are successively less so.

When comparing the cross section after the compression test on the spatterdash layer (Figure 8) with that of the top coat 


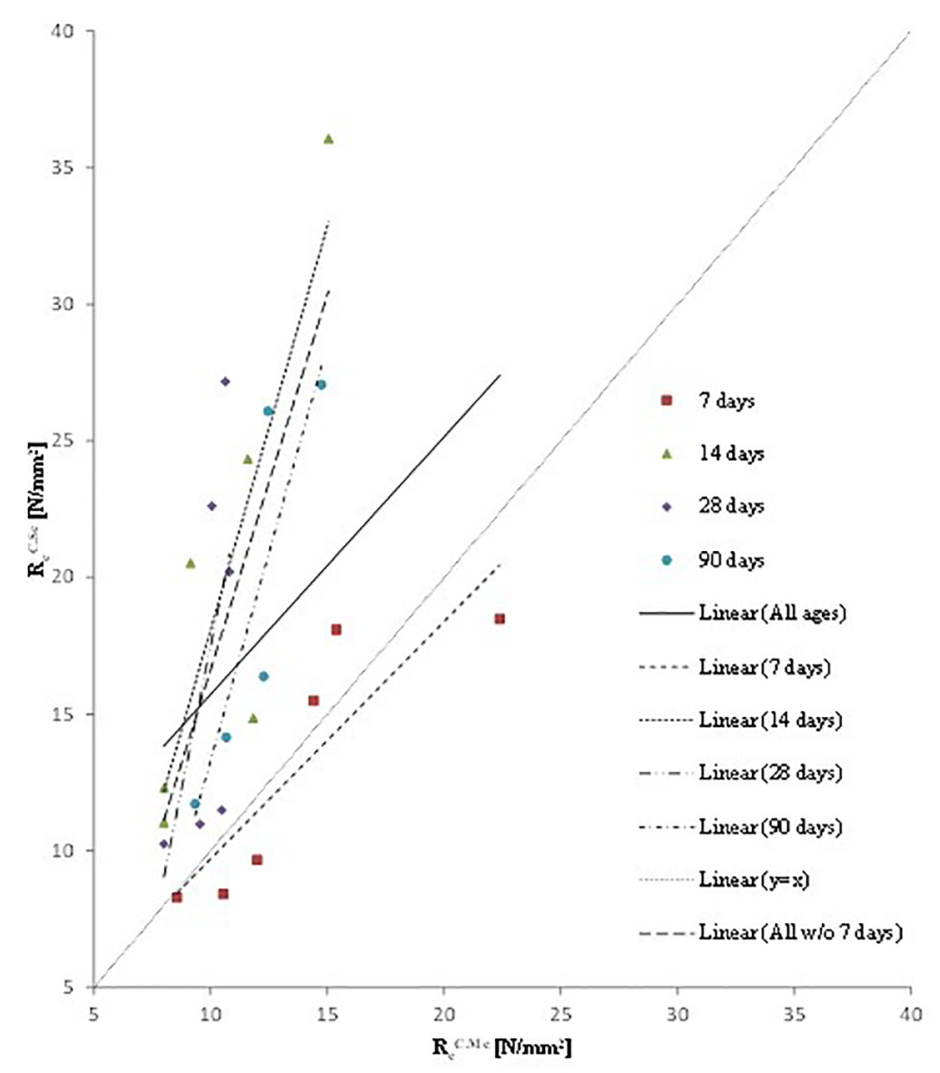

\begin{tabular}{|c|c|c|}
\hline Age & Relation & Correlation coefficient \\
\hline All & $R_{c}^{C . S c}=0.9432 \times R_{c}^{C . M c}+6.2931$ & $R^{2}=0.1754$ \\
\hline 7 days & $R_{c}^{C . S c}=0.8688 \times R_{c}^{C . M c}+1.0068$ & $R^{2}=0.7618$ \\
\hline 14 days & $R_{c}^{C . S c}=2.9561 \times R_{c}^{C . M c}-11.4990$ & $R^{2}=0.7472$ \\
\hline 28 days & $R_{c}^{C . S c}=4.2013 \times R_{c}^{C . M c}-24.5590$ & $R^{2}=0.3755$ \\
\hline 90 days & $R_{c}^{C . S c}=3.0372 \times R_{c}^{C . M c}-17.0730$ & $R^{2}=0.7733$ \\
\hline All except 7 days & $R_{c}^{C . S c}=2.7445 \times R_{c}^{C . M c}-10.8310$ & $R^{2}=0.5789$ \\
\hline
\end{tabular}

Caption: $\mathbf{R}_{\mathbf{c}}{ }^{\text {C.Mc }}$ - compressive strength of multi-coat traditional render; $\mathbf{R}_{\mathbf{c}}{ }^{\mathrm{C} . S c}$ - compressive

\section{strength of single-coat traditional render}

Figure 6. Relationship between the strength of multi-coat and single-coat cores of tradi-tional render.

(Figure 8, right) we can see that the spatterdash layer does not suffer significant degradation under the compressive strength test, noting that this is a square cross section, while the top coat suffers greater degradation and loss of its cross section area, which indicates that it was predominant in the failure mechanism during the test.

A small comparative analysis of the multilayer specimens, with and without spatterdash was possible in the compressive strength test at 90 days (Figure 9).
It was noted that the strength of the two-coat (base coat and top coat) specimens is slightly higher than that of the three-coat ones, as shown in Figure 9, with a change in the trend line slopes of about $0.62(0.62=2.18-1.56)$, which results in a difference of $2.8 \mathrm{~N} / \mathrm{mm}^{2}$ in comparison with higher value strengths (worse case). Where strengths are lower, the difference between them in the specimens with and without spatterdash is about $1 \mathrm{~N} / \mathrm{mm}^{2}$, which supports the provision in EN 13914- $1^{5}$ whereby spatterdash is not regarded as a coating layer. 


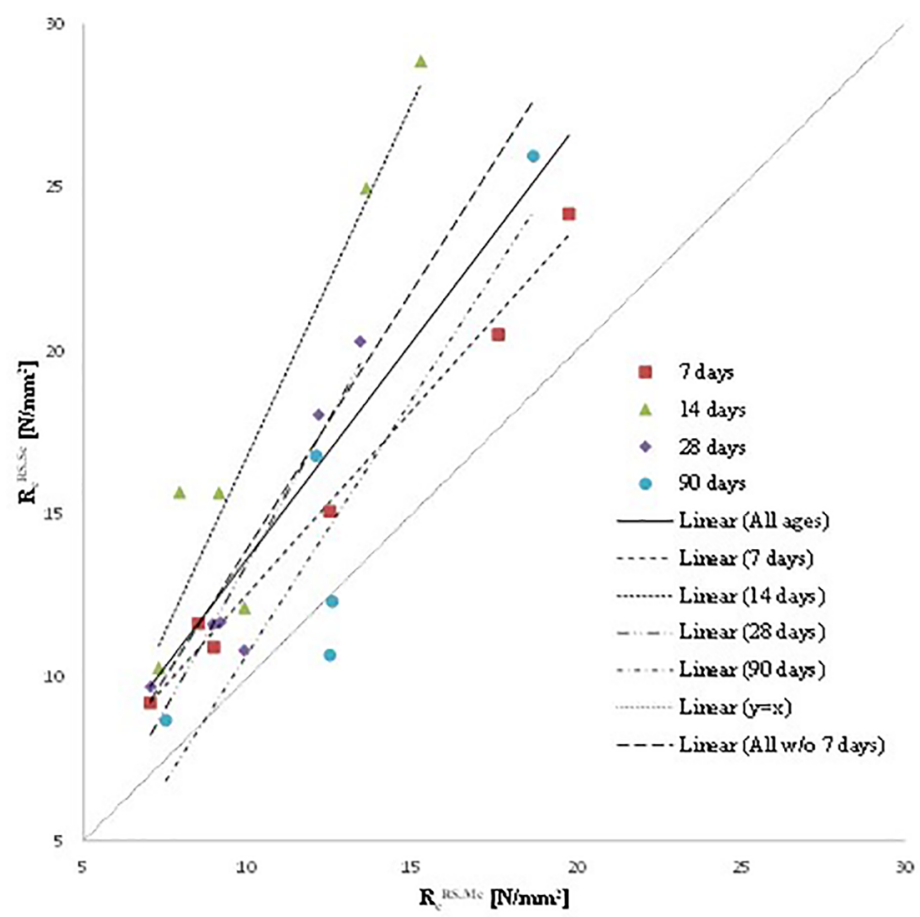

\begin{tabular}{|c|l|c|}
\hline Age & Relation & Correlation coefficient \\
\hline All & $R_{c}^{R S . S c}=1.3237 \times R_{c}^{R S . M c}+0.4203$ & $R^{2}=0.6858$ \\
\hline 7 days & $R_{c}^{R S . S c}=1.1231 \times R_{c}^{R S . M c}+1.3357$ & $R^{2}=0.9902$ \\
\hline 14 days & $R_{c}^{R S . S c}=2.1516 \times R_{c}^{R S . M c}-4.7022$ & $R^{2}=0.8800$ \\
\hline 28 days & $R_{c}^{R S . S c}=1.7808 \times R_{c}^{R S . M c}-4.2941$ & $R^{2}=0,9012$ \\
\hline 90 days & $R_{c}^{R S . S c}=1.5574 \times R_{c}^{R S . M c}-4.8393$ & $R^{2}=0.8126$ \\
\hline All except 7 days & $R_{c}^{R S . S c}=1.5754 \times R_{c}^{R S . M c}-1.7939$ & $R^{2}=0.6569$ \\
\hline
\end{tabular}

Caption: $\mathbf{R}_{\mathbf{c}}{ }^{\mathrm{RS} . M c}$ - compressive strength of multi-coat traditional render; $\mathbf{R}_{\mathbf{c}}{ }^{\mathrm{RS} . \mathrm{Sc}}$ - compressive strength of single-coat traditional render

Figure 7. Relationship between the strength of multi-coat and single-coat reference specimens of traditional render
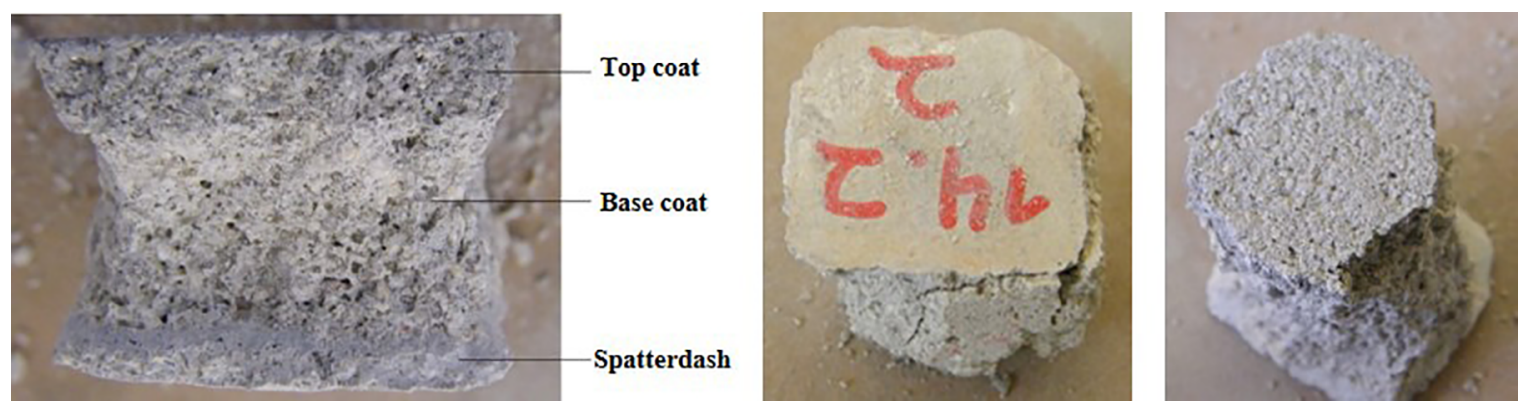

Figure 8. Square cross section reference specimen of multi-coat traditional render after compressive strength test (profile view, left; base of spatterdash layer, centre; surface of top coat, right). 


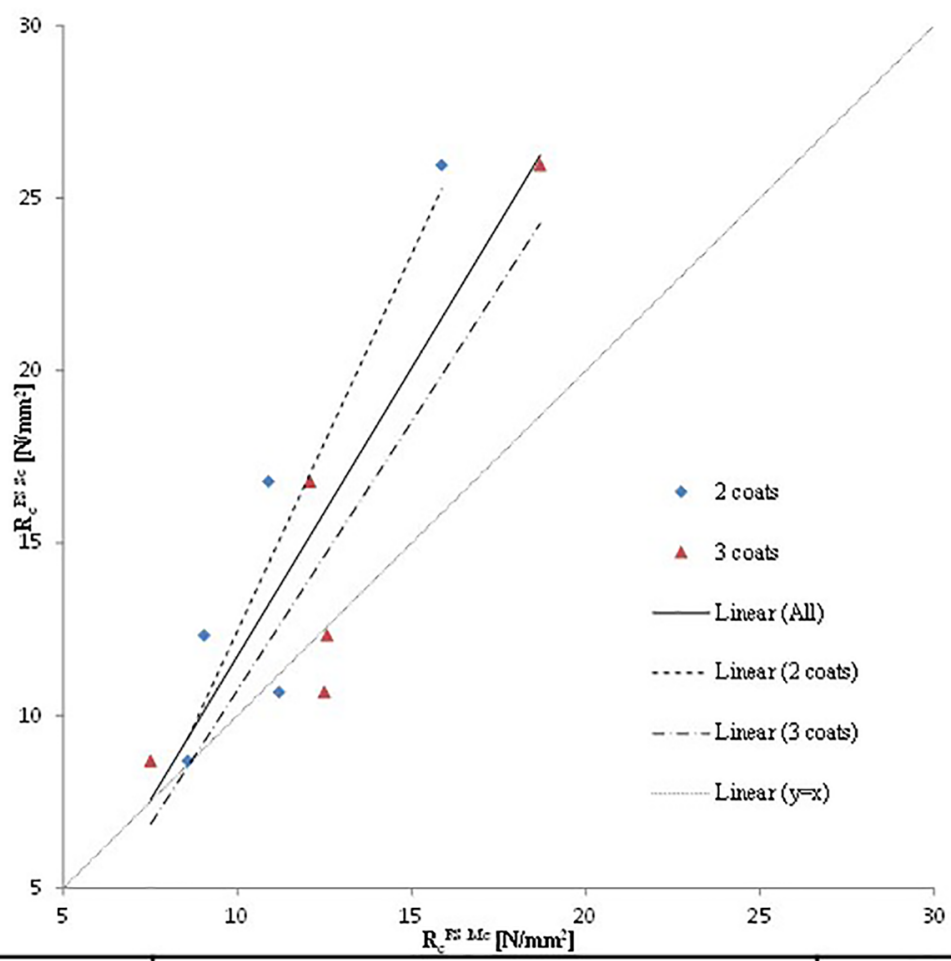

\begin{tabular}{|c|l|c|}
\hline $\begin{array}{c}\text { Distinction by number } \\
\text { of render coats }\end{array}$ & Relation & Correlation coefficient \\
\hline All & $R_{c}^{R S . S c}=1.6695 \times R_{c}^{R S . M c}-4.9614$ & $R^{2}=0.7576$ \\
\hline 2 coats & $R_{c}^{R S . S c}=2.1847 \times R_{c}^{R S . M c}-9.3876$ & $R^{2}=0.8428$ \\
\hline 3 coats & $R_{c}^{R S . S c}=1.5574 \times R_{c}^{R S . M c}-4.8393$ & $R^{2}=0.8126$ \\
\hline
\end{tabular}

Caption: $\mathbf{R}_{c}{ }^{\text {RS.Mc }}$ - compressive strength of multi-coat traditional render; $\mathbf{R}_{c}{ }^{\text {RS.Sc }}$ - compressive

strength of single-coat traditional render; 2 coats - two-coat traditional render with base and top

coats; 3 coats - traditional render with spatterdash, base and top coats

Figure 9. Relationship between strengths of reference specimens of multi-coat and sin-gle-coat traditional render at 90 days of age.

\subsection{Compressive strength relationship between multi-coat cores and single-coat reference specimens}

Despite the acceptable correlations found from the analysis of the relationship between multi-coat traditional render and single-coat for Cs and RSs, treated separately, it was not possible to relate multi-coat traditional render cores with single-coat traditional reference specimens, as we can see in Figure 10, where the best relationship occurs for the 7-day results (no top coat) with a slope of 0.94 (close to y $=\mathrm{x})$, with a satisfactory correlation coefficient $\left(\mathrm{R}^{2} \approx 0.59\right)$.
So it has been an indication that there is a direct relationship between the strength determined with multi-coat traditional render and that found with single-coat traditional render (produced with the same mix and layer size as the multi-coat traditional base coat render). But it has only been possible to establish a relationship between the strengths obtained with single-coat render specimens and multi-coat render cores aged 7 days (where the traditional render cores do not have a top coat).

So a more detailed study on this issue is needed to establish a relationship associated with having a top coat, since this layer has a strong impact on compressive strength. For this 


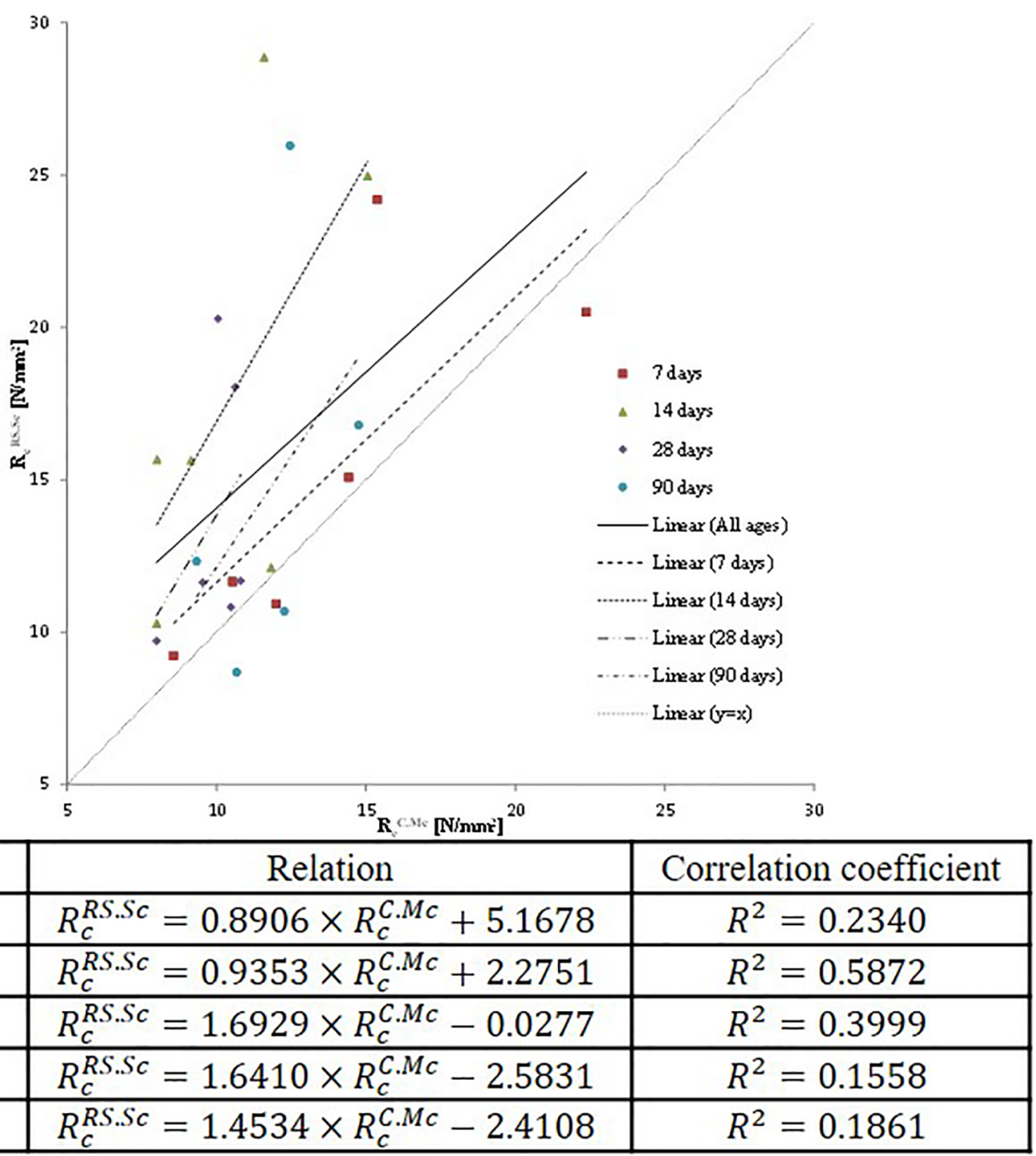

Caption: $\mathbf{R}_{\mathbf{c}}{ }^{\mathrm{C} . \mathrm{Mc}}$ - compressive strength of multi-coat traditional render cores; $\mathbf{R}_{\mathrm{c}}{ }^{\mathrm{RS}} \mathrm{Sc}$

\section{- compressive strength of reference specimens of single-coat traditional render}

Figure 10. Relationship between the strength of multi-coat cores and reference speci-mens of a single-coat traditional render.

purpose, the performance of individual tests for each layer is necessary, varying cement contents and thicknesses. In addition it is important to carry out auxiliary tests, such as: the elasticity modulus and Poisson measurements. Then, the comparison of the previous experimental results with a finite element analysis should be followed to study in depth the effect of the top coat on the compressive strength value.

\subsection{The contribution of each coat to the multi- coat render}

The results enable the assessment of the contribution of the different layers of multi-coat renders to overall compressive strength.

It is concluded that the first coat, the spatterdash does not significantly influence compressive strength. The results listed below enable this last conclusion to be drawn:

- At 7 days of age (multi-coat system consisting of spatterdash and base coat) - compressive strength values are very close to those obtained with singlecoat traditional render (same constitution as the multilayer render's base coat); 
- Comparison of a multi-coat render (spatterdash, base coat and top coat) with a two-layer one (base coat and top coat) at 90 days of age did not find any significant differences in the compressive strength values,

- In practical application onsite the spatterdash's thickness is not uniform and its contribution to the analysis of compressive strength can be disregarded.

Meanwhile, the third layer, the top coat, had a strong influence on the compressive test value for multi-coat renders. It was found that this layer suffered greater degradation in the compressive strength test (with a greater loss of area than occurred with the spatterdash) and therefore further investigation is needed.

The results at 90 days presented an $\mathrm{R}^{2} \geq 0.77$ and showed a slope near to those obtained at 14 and 28 days, indicating a stable trend with the age of the render, which is useful since in-service performance tests are commonly performed on existing buildings.

\section{Conclusions}

This paper discussed alternative methods to better characterize the compressive strength of multi-coat renders on in-service building façades, instead of using standard lab specimens with a $40 \mathrm{~mm}$ thick single-coat mortar. The experimental results have shown that the global compressive strength of a multi-coat render depends on the number and type of coats:

- The spatterdash (the coat with the highest cement content) does not significantly influence the compressive strength results and has a non-uniform thickness;

- The base coat thickness seems not to affect the relationship between multi-coat and single-coat specimens (as long as the thickness of the base coat is similar in the two types of specimens);

- The top coat (the coat with lowest cement content) has a high influence on the multi-coat mortar compressive strength, showing less compressive strength than the respective single-coat render (slope $>1$ ). Therefore the contribution of this coat should be taken into account in the interpretation of in-service compressive strength results.

In this context, two alternative methods can be used to assess the in-service mechanical performance of multi-coat renders: 1) to produce lab specimens with the same layers, that can be difficult to represent exactly the applied render, without the substrate interaction; or 2) collect cores from on-site testing, for example after a pull-off test. The use of cores takes into account the real in-service conditions (the influence of the substrate; the degradation agents' action; the application process; among others) and is useful in comparative assessments, for example: to compare the mechanical behaviour of a multi-coat rendering mortar on a certain façade (including damage and non-damage areas). The core experimental results also showed a high variability with lower correlations than the ones observed in reference specimens, due to several factors, such as: the difference on sizes and slenderness of the cores, the possible microcracking damage during cores extraction; or the water suction of the substrate. However, cores represent the real in-service conditions and minimize the use of complex numerical methods; their combination with other in-situ techniques (to measure indirectly mechanical characteristics and pulloff as a probe technique) reduces the results variability and increase the accuracy of the multi-coat diagnosis based on cores assessment.

Future experimental work should try to establish a relationship between the compressive strength of a multi-coat render and various formulations of the top coat. Also, the influence of each coat, with various thickness and formulations, should be detailed by further development. These experimental studies can support numerical relationships between the contributions of each coat to the overall of multilayer render compressive strength.

\section{References}

1. CSN EN 13791 - Assessment of in-situ compressive strength in structures and pre-cast concrete components, 2007. Brussels, Belgium, 2005.

2. Concrete Society - Concrete core testing for strength. Technical report 11, London, the Concrete Society, 1976.

3. CEN EN 998-1: 2010 - Specification for mortar. Rendering and plastering mortar. Brussels, Belgium, 2010.

4. CEN EN 1015-11:1999 (Ed.1) - Methods of test for mortar for masonry. Part 11: Determination of flexural and compressive strength of hardened mortar. Brussels, Bel-gium, 1999.

5. CEN EN 13914-1:2005 (Ed. 1) - Design, preparation and application of external rendering and plastering - External rendering. Brussels, Belgium, 2005.

6. Gomes R, Veiga M, Brito J. Influence of execution procedures on current renders (in Portuguese). 1st National Congress on Construction Mortars : APFAC, 2005. http://www.apfac.pt/ congresso2005/comunicacoes/Paper\%2021.pdf

7. Válek, J., Veiga, M. - Characterisation of mechanical properties of historic mortars - testing of irregular samples. Structural Studies, Repairs and Maintenance of Heritage Architecture IX. WIT Press 83, 2005, pp. 365 - 374.

8. Magalhães A, Veiga M.. Physical and mechanical characterisation of historic mortars. Application to the evaluation of the state of conservation. Materiales de Construcción. 2009;59(295):61-77.

9. Indelicato, F. - Estimate of concrete cube strength by means of different diameter cores: A statistical approach. Materials and Structures, Volume 33, Issue 3, 1997, pp. 131-138.

10. Flores-Colen, I. - Evaluation methodology of the in-service performance of façades from the predictive maintenance standpoint (in Portuguese), PhD Thesis on Civil Engi-neering, Technical University of Lisbon, Lisbon, Portugal, 2009, 487 p. 
11. Bazant, Z. P. - Size effect in blunt fracture, concrete, rock, metal. Journal of Engi-neering Mechanics, Volume 110, Issue 4, 1984, pp. 518-535.

12. Bazant, Z., Xi, Y., Reid, S. G. - Statistical size effect in quasibrittle structures: I. Is Weibull theory applicable? Journal of Engineering Mechanics, Volume 117, Issue 11, 1991, pp. 2609-2622.

13. Majeed, S. - Effect of specimen size on compressive, modulus of rupture and split-ting strength of cement mortar. Journal of Applied Sciences, Volume 11, Issue 3, 2011, pp. 584-588.

14. ASTM C42 - Standard method of obtaining and testing drilled cores and sawn beams of concrete, PA, USA, 1992.

15. Gonnerman, H. - Effect of size and shape of test specimen on compressive strength of concrete. Philadelphia: ASTM Proc. 25, Part. II, 1925, pp. 237-250.

16. Drdácký, M., Masín, D., Mekonome, M.D., Slízková, Z. Compression testes on non-standard historic mortar specimens, HMC08 - Historical Mortars Conference, LNEC, Lisbon, 2008, CD

17. Benedetti, A., Luca, P. - Experimental characterization of mortar by testing on small specimens. 15th International Brick and Block Masonry Conference. Florianópolis, Brazil, 2012, CD.
18. Soares, A., Flores-Colen, I., de Brito, J. - Influence of slenderness on the compres-sive strength evaluation of cores of renders. Materials and Structures, Volume 48, Issue 5, 2015, pp. 1449-1460.

19. Soares, A., Flores-Colen, I., de Brito, J. - The use of non-standard specimens to study the compressive strength of multi-coat renders. Journal of Testing and Evaluation, Volume 43, Issue 5, 2015, pp. 1055-1065.

20. CEN EN 196-1 - Methods of testing cement. Part 1: Determination of strength. Brussels, Belgium, 2005.

21. CEN EN 1015-2:1998 (Ed.1) - Methods of test for mortar for masonry. Part 2: Bulk sampling of mortars and preparation of test mortars". Brussels, Belgium, 1998

22. European Committee for Standardization. European Standard - EN 1015-12:2000 (Ed.1) - Methods of test for mortar for masonry. Part 12: Determination of adhesive strength of hardened rendering and plastering mortars on substrates. Brussels: European Committee for Standardization; 2000.

23. RILEM - MDT.D.3 Determination "in situ" of the adhesive strength of rendering and plastering mortars to their substrate. RILEM Recommendation, Materials and Struc-tures, Volume 37, Issue 7, 2004, pp. 488-490. 